\title{
Household Contact
}

National Cancer Institute

\section{Source}

National Cancer Institute. Household Contact. NCI Thesaurus. Code C102646.

A way of contracting a disease through interactions with an infected person in the same residence. 